\title{
Corneal Perforation in a Patient with History of Unidentified Urethritis
}

\author{
Glenda Espinosa-Barberi1 ${ }^{*}$, Sara Miranda Fernández1, Rosa Borges Trujillo1, \\ Joaquín José Rutllán Civit' ${ }^{1}$ Iballa Horcajada Herrera²
}

${ }^{1}$ Department of Ophthalmology, Hospital Universitario de Gran Canaria Doctor Negrín, Las Palmas de Gran Canaria, Spain ${ }^{2}$ Department of Microbiology, Hospital Universitario de Gran Canaria Doctor Negrín, Las Palmas de Gran Canaria, Spain Email: ^glenda_eb@hotmail.com

How to cite this paper: Espinosa-Barberi, G., Fernández, S.M., Trujillo, R.B., Civit, J.J.R. and Herrera, I.H. (2018) Corneal Perforation in a Patient with History of Unidentified Urethritis. Open Journal of Ophthalmology, 8, 36-41.

https://doi.org/10.4236/ojoph.2018.81006

Received: December 12, 2017

Accepted: February 4, 2018

Published: February 7, 2018

Copyright $\odot 2018$ by authors and Scientific Research Publishing Inc. This work is licensed under the Creative Commons Attribution International License (CC BY 4.0).

http://creativecommons.org/licenses/by/4.0/

\begin{abstract}
Purpose: A 40-year-old male with a severe corneal melting, peripheral infiltration and nasal perforation in the right eye after a not affiliated case of urethritis is reported. Methods: Retrospective case report. Results: A sample of conjunctival discharge was sent to culture, in where Gram-diplococci grew and therefore a systemic antibiotic treatment was initiated. Given the severity of the clinic, a penetrating keratoplasty is conducted two days after admission and the corneal button was sent to microbiology, which polymerase chain reaction was positive for Neisseria gonorrhoeae. Conclusion: Despite it being a rare process in an adult, keratitis caused by Neisseria gonorrhoeae should be suspected in all sexually active patients with conjunctivitis and with a clinic genitourinary medical history. The systemic therapy showed a superior effectiveness in combination with topical therapy than the last one on its own.
\end{abstract}

\section{Keywords}

Corneal Perforation, Gonococcal Conjunctivitis, Keratoconjunctivitis, Microbiological Culture

\section{Introduction}

Neisseria gonorrhoeae is a gram-aerobic diplococci which could take two different clinical forms, one of them in neonates and the other in sexually active adults [1]. Although it is one of the most common causes of sexually transmitted disease, eye infection is uncommon, especially in adults, and it should be considered a medical emergency. Gonococcal infection incidence has decreased between the ages of 70 and 80 as a consequence of the vaccine emergence. However, 
over the last few years it has increased and it is believed to have happened due to the neglect of the barrier method use [2].

The eye clinical picture is presented as a potentially destructive hyperacute purulent keratoconjunctivitis, which clinical diagnosis is complex as it could cause some confusion with other malfunctions which are overinfected adenoviral conjunctivitis and autoimmune processes with marginal keratolysis [3]. Due to the increasing antiobiotics resistance, the guidelines of the treatment have been modified. A wrong or late treatment could have serious visual distortion consequences to the affected eye.

\section{Case Report}

A 40-year-old male is sent due to a corneal melting process in his right eye (RE) after suffering urethritis two weeks before, which was treated successfully with azithromycin (400 mg/day) for 3 days. The patient was sent with a case of conjunctivitis with abundant purulent discharge which was previously treated with topical tobramycin and dexamethasone, with symptomatic aggravation. In his background, he is allergic to codeine, streptomycin and aspirin and has been an occasional consumer of cannabis and cocaine, in addition to having risk sex previously. The visual acuity (VA) in his RE was of luminous perception, while the one in his left eye (LE) was the unit.

The examination of the anterior segment showed a conjunctival chemosis, a diffuse and severe corneal thinning, dense annular infiltration and nasal microperforations occluded by the iris (Figure 1). The eye was in athalamia. Conjunctival secretions samples are sent for the culture and Gram. The intravenous therapy is introduced with piperacillin every 6 hours, ceftriaxone every 12 hours and vancomycin and ceftazidime reinforced eye drops.

The result of the culture was negative, while Gram-diplococci grew (Figure $2(\mathrm{~A}))$. The genital examination and serology were normal. Due to the gravity of this case, the negativity of the cultures and the condition of the cornea, a penetrating keratoplasty is conducted two days later and the button is sent to the department of microbiology.

The polymerase chain reaction (PCR) was positive for Neisseria gonorrhoeae (Figure 2(B)), without identifying other concomitant germs. The diagnosis of corneal perforation was then confirmed as a complication of conjunctivitis of gonococcal origin. The patient's ocular tissues have been infected by contact with the urethral secretions themselves through unhygienic hands, and the ophthalmologic clinic appeared between 1 - 2 weeks after urethritis. Subsequently the conjunctivitis not being treated correctly resulted in the corneal perforation thanks to the proteolytic activity of the bacterium.

Treatment with doxycycline $100 \mathrm{mg}$ every 12 hours is added. Eleven months after surgery, the graft is totally transparent, anterior chamber details can be correctly seen, the VA is of luminous perception and colors (due to the development of a cataract) and the intraocular pressure is $11 \mathrm{~mm} \mathrm{Hg}$ (Figure 3). 


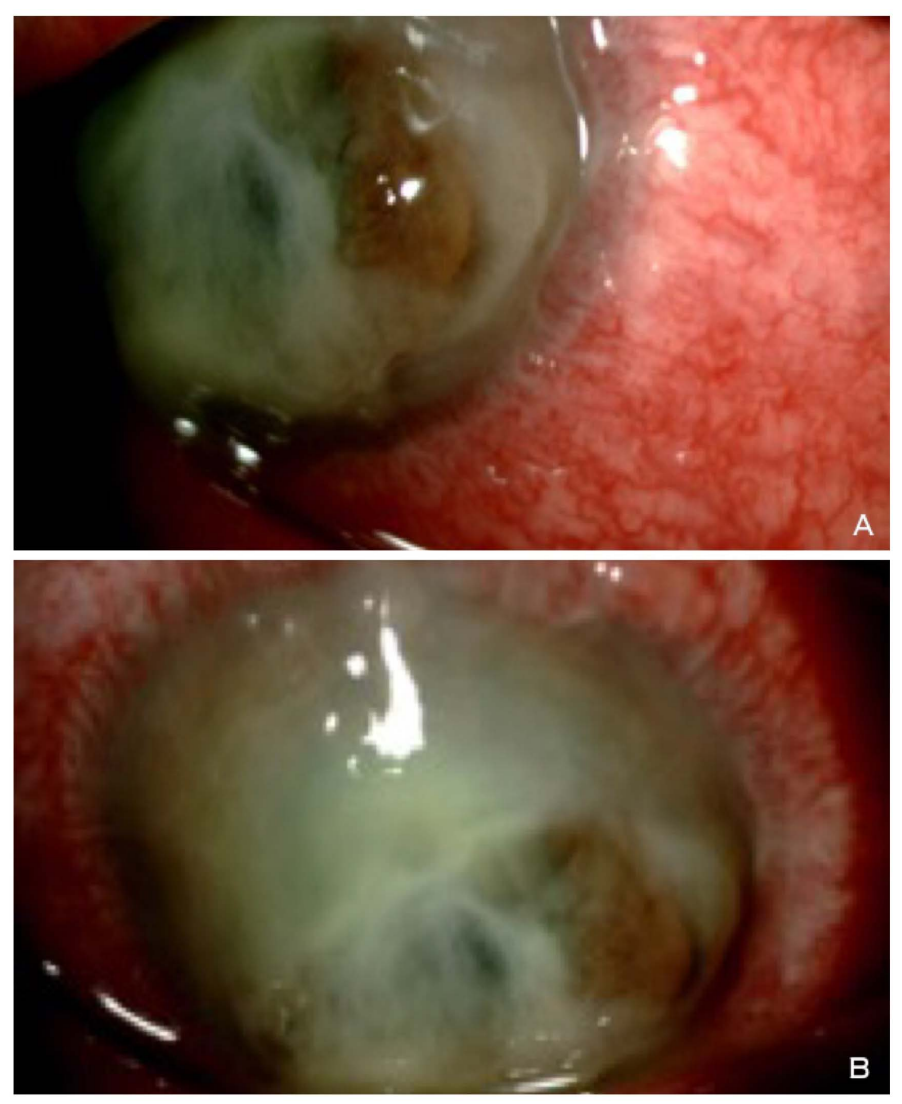

Figure 1. (A)-(B): Slit lamp. Nasal corneal perforation blocked by iris with infiltration and suppuration in the right eye.

\section{Discussion}

Most of the Neisseria species are colonizers of the nasopharyngeal mucosa. Both Neisseria gonorrhoeae and meningitidis are considered as pathogens for humans. Neisseria gonorrhoeae is able to produce proteolytic enzymes, which give it the ability of penetrating the intact corneal epithelium, therefore, present a high virulence and destructive ability of the affected tissues. Due to the fast evolution towards the corneal thinning a few days after having acquired the infection, the clinical picture is to be considered an ophthalmologic emergency. Genitourinary symptoms could precede the eye once in one or two weeks [4] [5].

Keratoconjunctivitis transmission in the adult is related to the direct contact of the conjunctiva with infected genitourinary secretions which seems to be the mechanism that occurred in our case. Despite this, cases produced by indirect contact with fomites have been identified and in low socioeconomic levels associated with inadequate hygiene [4] [6].

It involves a rare entity in an adult, but there has been an increase of its incidence over the last few years, which coincides with other sexually transmitted diseases, it is therefore necessary to conduct a screening, as done in our case. The coinfection with Chlamydia trachomatis is between $30 \%-40 \%$ of the cases [4] [5] [6]. 

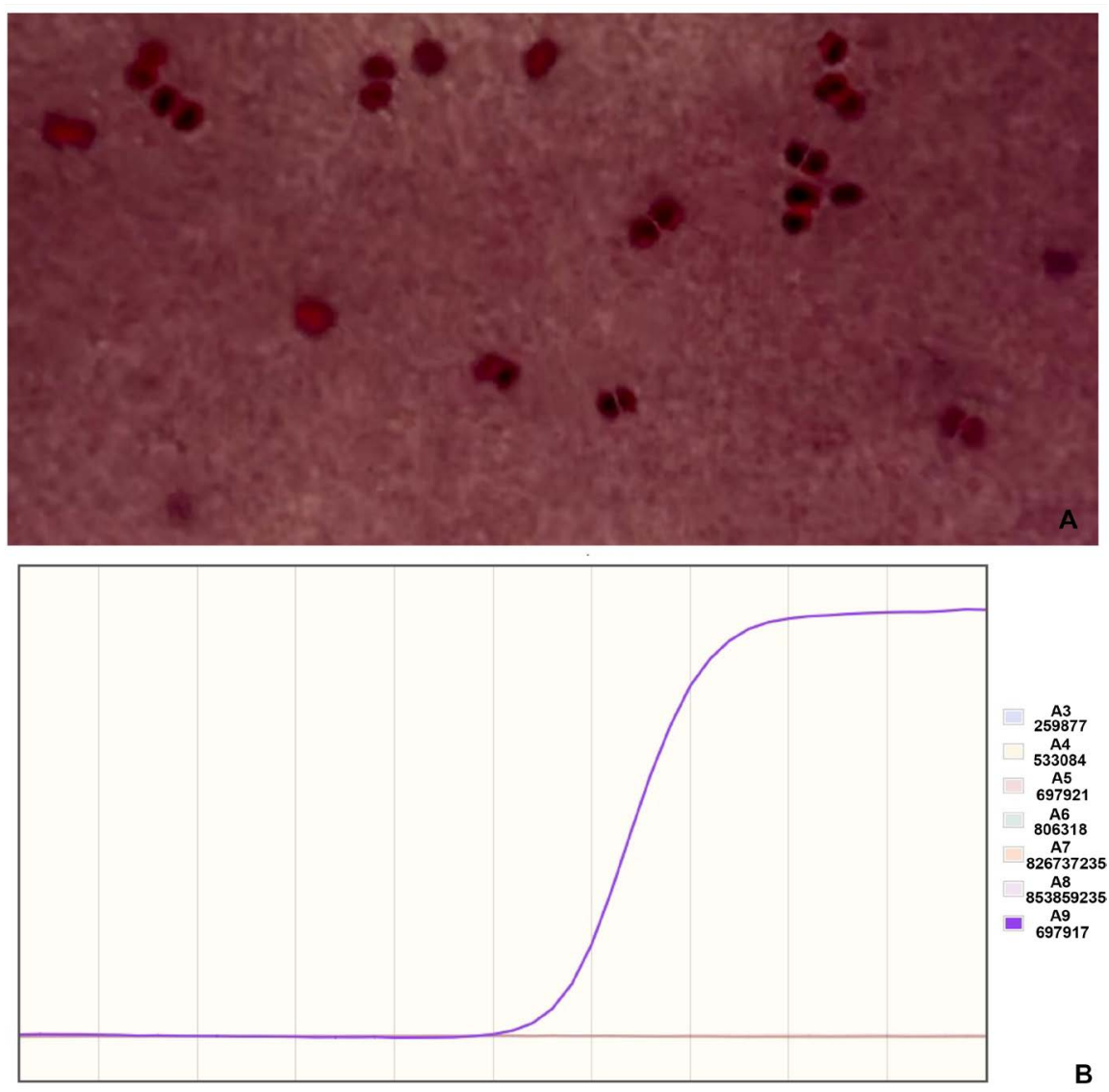

Figure 2. (A) Gram stain in a sample of conjunctival secretions. Gram-dipococci are seen facing each other, with the typical appearance of "coffee beans"; (B) PCR amplification curve in real-time. An amplification curve for Neisseria gonorrhoeae in a sample of corneal button is produced with a threshold cycle with a valid range of result.

The most common clinical signs are erythema and palpebral edema, conjunctival injection and hyperacute purulent discharge, melting and corneal perforation. To confirm the diagnosis a Gram stain must be done in order to detect intracellular Gram-diplococci and culture in enriched media like chocolate agar or modified Thayer-Martin.

The PCR is a rapid diagnostic method, with a high sensitivity and specificity (greater than 95\%) compared to culture [7] reflecting the greater tolerance of deficiencies in the process of collection, transport and conservation of the samples. There is not authorized nucleic acid amplification test that can be performed with extragenital samples, but there are increasing indications that these are more sensitive than the culture for these locations [8]. Since PCR has been positive at first, it is not necessary, in these cases of gonococcal infection, to perfom it more often, since it is a test with high sensitivity and specificity, so in combination with de clinic that the patient presented and the Gram staining, is considered confirmatory. In the routine, quantitative gonococcal PCR is not performed, since the searched result is qualitative (positive or negative, not as in the case of human immunodeficiency virus infection, for example, where copies $/ \mathrm{ml}$ must be quantified, to determine the viral load). 

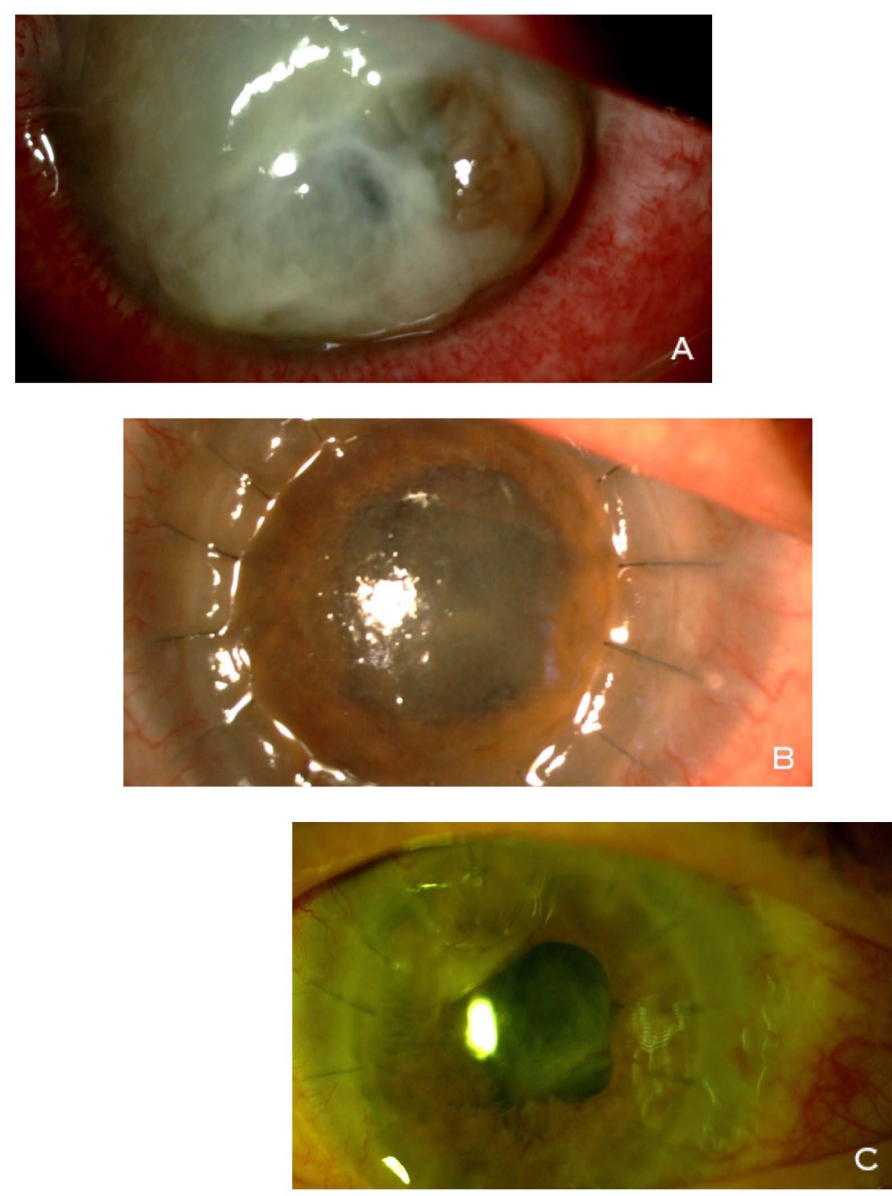

Figure 3. Slit lamp (A) before surgery, (B) one month after where it is appreciated a corneal clearance and formed anterior chamber and $(C)$ six months after surgery the cornea is totally transparent with less inflammation.

The current treatment is done with intramuscular ceftriaxone, associated with topical treatments that contain erythromycin, quinolone, etc., which has proved to have a superior efficiency than topical treatment in isolation. In corneal melting cases where there is no correct response to treatment and the perforation risk is imminent, or has already been produced, keratoplasty has proven to be the most efficient invasive therapy and could therefore restore the globe in its whole. It could also reduce the risk of endophthalmitis, if it used at an early stage as in our case [9] [10].

\section{Conclusion}

In conclusion, gonococcal conjunctivitis is a rare entity that should not be forgotten. The evolution of the disease without treatment is an important risk factor in the complications generated and in the visual prognosis of the patient. The PCR of ocular samples, in addition to the clinical and Gram staining, provides diagnostic value in those cases where the culture is not conclusive for various reasons, as in the one previously presented. 


\section{Disclosure}

The authors report no disclosures relevant to the manuscript.

\section{References}

[1] Sparling, P.F. and Handsfield, H.H. (2000) Neisseria gonorrhoeae. Principles and Practice of Infectious Diseases. In: Mandell, Douglas and Bennet, 5th Edition, Churchill Livingstone, New York.

[2] Little, J.W. (2006) Gonorrhea: Update. Oral Surgery, Oral Medicine, Oral Pathology, Oral Radiology, and Endodontology, 101, 137-143. https://doi.org/10.1016/j.tripleo.2005.05.077

[3] Lee, J., Choi, H., Lee, J., et al. (2002) Gonococcal kerato Conjunctivitis in Adults. Eye, 16, 646-649. https://doi.org/10.1038/sj.eye.6700112

[4] Wan, W.L., Farkas, G.C., May, W.N., et al. (1986) The Clinical Characteristics and Course of Adults Gonococcal Conjunctivitis. American Journal of Ophthalmology, 102, 575-583. https://doi.org/10.1016/0002-9394(86)90527-1

[5] Schwab, L. and Tizazu, T. (1985) Destructive Epidemic Neisseria gonorrhoeae Keratoconjunctivitis in African Adults. British Journal of Ophthalmology, 69, 525-528. https://doi.org/10.1136/bjo.69.7.525

[6] Ullmann, S., Russell, T.J. and Forster, R.K. (1987) Gonococcal kerato Conjunctivitis. Survey of Ophthalmology, 32, 199-208. https://doi.org/10.1016/0039-6257(87)90095-6

[7] Leber, A.L., May, G.S. and Le Bar, W.D. (2006) Nucleic Acid Amplification Tests for Detection of Chlamydia trachomatis and Neisseria gonorrhoeae. Cumitech 44, Washington DC.

[8] Bahman, L.H., et al. (2010) Nucleic Acid Amplification Tests for Diagnosis of Neisseria gonorrhoeae and Chlamydia trachomatis Rectal Infections. Journal of Clinical Microbiology, 48, 1827-1832. https://doi.org/10.1128/JCM.02398-09

[9] Newman, L.M., Moran, J.S. and Workowski, K.A. (2007) Update in the Management of Gonorrhea in Adults in the United States. Clinical Infectious Diseases, 44, 84-101. https://doi.org/10.1086/511422

[10] Nurozler, A.B., Salvarli, S.I., Budak, K., et al. (2004) Results of Therapeutic Penetrating Keratoplasty. Japanese Journal of Ophthalmology, 48, 368-371. https://doi.org/10.1007/s10384-004-0083-x 